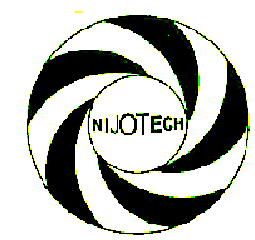

Nigerian Journal of Technology (NIJOTECH)

Vol. 33. No. 3, July 2014, pp. $324-\mathbf{3 3 0}$

Copyright@ Faculty of Engineering,

University of Nigeria, Nsukka, ISSN: $1115-8443$

www.nijotech.com

http://dx.doi.org/10.4314/njt.v33i3.9

\title{
PERFORMANCE OPTIMIZATION OF A CASSAVA PELLETING MACHINE
}

\author{
C. H. Kadurumba* \\ NATIONAL Root Crops ReSEARCH InSTITUTE UMUdike, ABIA STATE. NIGERIA \\ Email address: kaduruchuma@yahoo.com
}

\begin{abstract}
Cassava pelleting in most developing countries are usually done manually. This process is very laborious, time consuming and unhygienic. A pelleting machine has been recommended because of its low cost, high capacity of providing pelleted cassava as well as relative ease of operation and maintenance. The machine consists of a screw conveyor for conveying the cassava mash through the dies openings, electric motor, pulley drives and belts. $A$ performance optimization of the system was carried out to maximize the solid product recovery, efficiency and throughput of the machine. The optimization results shows that the responses efficiency, throughput and power consumption of the machine were determined as $58 \%, 75.76 \mathrm{~kg} / \mathrm{hr}$ and $4106.36 \mathrm{~W}$ respectively. Mathematical models were developed using dimensional analysis. The independent variables such as screw pitch, conveyor speed and bulk density were used to derive the dimensionless parameters used as input variables for the regression analysis. The dependent variables like the mass number, power number and the processing time number were measured and compared to computed values such as their standard errors, percentage errors and coefficient of determination.
\end{abstract}

Keywords: pellets, screw conveyor, mass number, power number, processing time number, density number

\section{INTRODUCTION}

Cassava serves as the major source of carbohydrate in West Africa. In Nigeria the crops can be processed into gari, lafum, fufu, cassava chips and pellets. The cassava roots are also used for livestock and fish feed. The cassava roots are exported in chips or pellet forms. Pelleting is an extrusion process which shapes a plastic or a dough-like material by forcing it through a restriction or die. Examples of pelleted materials include, rolling of noodles and pie crust dough, finger stuffing of chopped meat through animal horns into natural casings, pressing of soft foods through hand ricers to produce string-like particles, and cranking of hand powered meat grinders. Pelleting machines are used to produce pellets. Cassava pellet is an unfermented dried cassava product obtained by compressing raw cassava chips under appropriate processing conditions resulting in the formation of dried bulky product suitable for animal feed and ease of transportation and exportation. Pelleting of cassava decreases the volume of cassava to be transported or exported by $25 \%$. This simplifies transport, handling and storage. It also produces uniform product which is less fragile for export than raw cassava chips. The first food extruders were based on the use of piston and ram. In Italy, single-screw extruders were used in the mid-1930s for pasta products. They employed low shear, deep flight screws and operated at low speeds [3]. The resulting products were not fully cooked. Moist dough was compressed by turning screw and shaped by the orifice through which the dough was expelled. In addition, these single-screwed Pelleting machines were very expensive and produced low tonnage of pellets. Later simple inexpensive extruders were developed in the United States in the 1960s foron-the farm cooking of soybeans, cereals and cassava feeds. This low cost extruder design was quickly adapted in the mid-1970s for use in nutrition intervention projects in many less developed countries [2]. In early 1980 the machine was modified and it became more reliable and was widely used to process different foods and crudely texturized food, though numerous mechanical problems were experienced. The objectives of this study are; (1) to develop a mathematical model for analyzing the machine using dimensional analysis. (2) to conduct a performance optimization of the machine. 


\subsection{MATERIALS AND METHODS}

\subsection{Machine Description and Evaluation of Parameters}

A photograph of the test machine is shown in figure 1 . In the housing a rotary supported screw conveyor is located. The cassava pelleting machine comprises of 3 main units. These include; the hopper, transmission and pelleting unit. The hopper is made of stainless material with its wall sloping at $62^{\circ}$. The transmission unit consists of a $30 \mathrm{~mm}$ shaft carrying the screw conveyor and it is supported in bottom and top bearings. The screw conveyor has varying pitch. The pitch of the screw conveyor at the inlet (i.e. directly below the opening where the hopper is mounted) is larger to maximize transportation of feedstock as it enters the barrel, while at the middle end (i.e. kneading and final cooking zone) the pitch is decreased so as to increase the temperature for cooking the pellets and increase the pressure for uniform forming of pellets in the die or extrusion plate. The die is attached to the end of the barrel by a circular flange point weld in place by five bolts so that the plate can be changed with other plates having different holes diameter for producing different sizes of pellets and for maintenance and cleaning of the machine. There is a knife arrangement at the face of the die that can be adjusted to cut pellets of different lengths as they are coming out of the machine. The entire machine is mounted on a frame made of $\mathrm{v}$ section (angle iron). The drive for the Pelleting machine is an electric motor of $4.125 \mathrm{~kW}$. The drive has a pulley which can hold two $\mathrm{V}$ - belts. The rotary motion of the drive is transferred from the pulley to the screw conveyor pulley. Vessels are used for collection of the products so that it can be utilized later.

\subsection{Determination of Dry Matter and Moisture Content}

Two grams of cassava mash each from twenty four different genotypes obtained from National Root Crops Research Institute Umudike were weighed into a separate crucible. They were heated for $24 \mathrm{hrs}$ in an oven at $100^{\circ} \mathrm{C}$, dried to a constant weight and cooled for ten minutes in desiccators. Afterwards they were weighed and the values were recorded. If the weight of cassava mash is $\mathrm{W}_{1}$, weight of empty crucible is $\mathrm{W}_{0}$, then the weight of crucible plus oven dried sample is $\mathrm{W}_{3}$. The equation for calculating the percentage dry matter and moisture content are,

$\%$ Dry matter $=\frac{\mathrm{W}_{3}-\mathrm{W}_{\mathrm{o}}}{\mathrm{W}_{1}-\mathrm{W}_{\mathrm{o}}} \times \frac{100}{1}$
$\%$ Moisture Content $=$

$100-\%$ Dry matter content

The dry matter content and moisture content were determined following the procedure of [1].

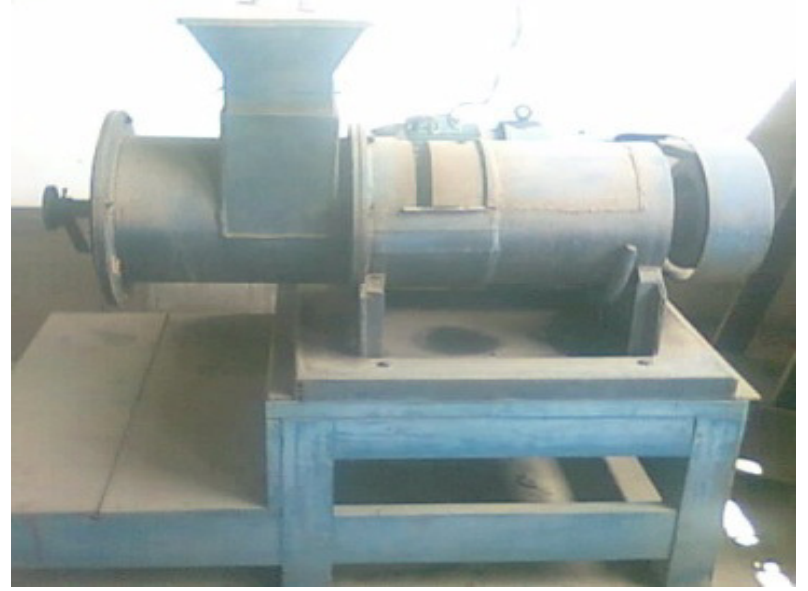

Figure 1: Photograph of the cassava pelleting machine.

\subsection{Determination of the Input Variables}

The starch content of the cassava genotypes was determined following the traditional method of starch extraction as described by [7]. $7 \mathrm{~kg}$ of freshly harvested cassava roots were peeled, washed in water and grated. The grated mash was sieved through a screen and suspended in a $0.01 \mathrm{~m}^{3}$ of water. This separates the fibrous and other coarse root material from the mash. The starch was allowed to settle for 4$6 \mathrm{hrs}$ before decanting. The supernatant was decanted and the thick sediment is the wet starch.

The starch was dried using a dryer at $60^{\circ} \mathrm{C}$ for $18 \mathrm{hrs}$ and packed. The values of screw pitch, helix angle, conveyor speed were selected to cover the expected operating range of the machine. To calculate the bulk density of the cassava mash, the following equation was used:

$$
\mathrm{B}_{\mathrm{c}}=\frac{\mathrm{W}_{2}-\mathrm{W}_{1}}{\mathrm{~V}}\left(\frac{\mathrm{kg}}{\mathrm{m} 3}\right)
$$

where $B_{c}$ is the bulk density of grated cassava mash in $\left(\mathrm{kg} / \mathrm{m}^{3}\right), \mathrm{W}_{2}$ is the weight of container + weight of sample in $(\mathrm{kg}), \mathrm{W}_{1}$ is the weight of container in $(\mathrm{kg})$ and $V$ is the volume of cylinder in $\left(\mathrm{m}^{3}\right)$

The process was repeated for about three times and the bulk density obtained. The cassava mash viscosity was measured using a cylindrical rotational viscometer which covers a temperature range from $30^{\circ} \mathrm{C}$ up to $150^{\circ} \mathrm{C}\left(-22^{\circ} \mathrm{F}\right.$ up to $\left.+302^{\circ} \mathrm{F}\right)$. The instrument consists of the control unit Visco-Temp 30150 with a cooling unit and sensors for measurement in low and medium viscosity ranges. 


\subsection{Model Development using Dimensional Analysis}

Buckingham Pi theorem $[4,5,6]$ was used in this study to derive equations and expressing one variable in terms of its dependent variables. It is commonly used when the number of variables exceeds four. Its application results in the formation of dimensionless parameters called the Pi ratios. The three fundamental dimensions commonly used for systems in mechanics are used. The basic dimensions are mass $\mathrm{M}$, length $\mathrm{L}$, and time $\mathrm{T}$. The number of dimensionless and independent quantities (Pi terms) required to fully express the relationship between the variables were determined as follows:

$$
S=n-b
$$

where, $\mathrm{S}$ is the number of Pi terms; $\mathrm{n}$ is the total number of variables and $b$ is the number of basic dimensions. The pertinent variables that affect the Pelleting machine performances are nine variables, (Table 1) with their corresponding dimensions.

Helix angle, Dry matter content, Moisture content, and starch content are dimensionless parameters. Dry matter content and starch content are linearly related to each other. Thus one of them (Dry matter content) was selected as characteristics of the cassava mash used in the study. Therefore, the number of dimensional variables is $n=7$, but since the basic dimensions are MLT $=3$, then, number of dimensionless $\pi$ - terms, $\quad \mathrm{S}=\mathrm{n}-\mathrm{b}, \mathrm{S}=7-3=4$

Hence, five Pi-terms say, $\pi_{1}, \pi_{2}, \pi_{3}$ and $\pi_{4}$ were generated. The repeating variables are

$B_{G}, B_{K}$ and $B_{D}$. The basic equations for each $\pi$ ratio derived are as follows:

$$
\begin{aligned}
\pi_{1} & =\frac{M_{o}}{D_{\rho}^{3}}=\text { Dimensionless Mass Number } \\
\pi_{2} & =\frac{P_{W}}{D^{5} C_{S}^{3} \rho}=\text { Dimensionless power number } \\
\pi_{3} & =\frac{\mu}{D^{2} C_{S} \rho}=\text { Dimensionless density number } \\
\pi_{4} & =\mathrm{tC}_{S}=\text { Dimensionless time number }
\end{aligned}
$$

In the dimensionless terms determination exercise, the two dimensionless terms; Helix angle $\left(\mathrm{H}_{\mathrm{A}}\right)$, and Dry matter content ( $\left.\mathrm{D}_{\mathrm{M}}\right)$ were excluded initially, but have to be added when the other dimensionless terms had been formed. They are represented as $\pi_{5}$ and $\pi_{6}$ respectively. The $\pi$-terms are formed based on the principle of dimensional homogeneity. The output $\pi-$ terms are related to the input $\pi$-terms as follows:

$$
Y=\theta\left(\pi_{3}, \pi_{5}, \pi_{6}\right)
$$

where $Y$ is any of the three (3) output or dependable variables $\pi_{3}, \pi_{5}$ and $\pi_{6}$

The constants a, b, c, and d are determined using the Minitab software.

Using a first order multivariate regression model, the output parameter is given as

$Y_{(i)}=a_{o}+a_{1} X_{1}+a_{2} X_{2}+a_{3} X_{3}$

where the coefficients $a_{\mathrm{i}}, \mathrm{i}=1,2 \ldots 3$ are determined by multiple regression.

The variables are given by the equations below:

$\mathrm{X}_{1}=\frac{\mu}{\mathrm{D}^{2} \mathrm{C}_{\mathrm{S}} \rho}$

$\mathrm{X}_{2}=\mathrm{H}_{\mathrm{A}}$

$\mathrm{X}_{3}=\mathrm{D}_{\mathrm{M}}$

Similarly, the output variables $Y_{i}$ are given by

$Y_{1}=\frac{M_{0}}{D^{3} \rho}$

$\mathrm{Y}_{2}=\frac{\mathrm{P}_{\mathrm{W}}}{\mathrm{D}^{5} \mathrm{C}_{\mathrm{S}}^{3} \rho}$

$\mathrm{Y}_{3}=\mathrm{tC}_{\mathrm{S}}$

If $\mathrm{R}^{2}>0.80, \mathrm{R}^{2}$ pred. $>0.80$ and $\mathrm{R}^{2}$ adj $>0.80$, the first order model may be considered sufficient for correlating the experimental results. A second order multivariate regression model may also be contemplated in case of minor improvements in the regression. The utilization of the second order regression model will only be necessary if a first-order model does not produce satisfactory results. We note that, from equations (5) to (8), the $\pi$-parameters already contain among them, various powers of the independent experimental variables listed in Table 1.

Table 1: Variables used in the Study and their Dimensions and Units.

\begin{tabular}{lccccc}
\hline \multicolumn{1}{c}{ Variables } & Type & Symbol & Unit & Dimensions & Number \\
\hline Mass of pellets formed & Dynamic & $\mathrm{M}_{\mathrm{o}}$ & $\mathrm{Kg}$ & $\mathrm{M}$ & $\mathrm{A}_{1}$ \\
Processing time & Kinematic & $\mathrm{t}$ & $\mathrm{S}$ & $\mathrm{T}$ & $\mathrm{A}_{4}$ \\
Power consumed & Dynamic & $\mathrm{PW}_{\mathrm{W}}$ & $\mathrm{kgm}^{2} / \mathrm{s}^{3}$ & $\mathrm{ML}^{2} \mathrm{~T}^{-3}$ & $\mathrm{~A}_{2}$ \\
Screw pitch & Geometric & $\mathrm{D}$ & $\mathrm{M}$ & $\mathrm{L}$ & $\mathrm{B}_{\mathrm{G}}$ \\
Conveyor speed & Kinematic & $\mathrm{C}$ & $\mathrm{rad} / \mathrm{s}$ & $\mathrm{T}^{-1}$ & $\mathrm{~B}_{\mathrm{k}}$ \\
Bulk density & Dynamic & $\rho$ & $\mathrm{Kg} / \mathrm{m}^{3}$ & $\mathrm{ML}^{-3}$ & $\mathrm{~B}_{\mathrm{D}}$ \\
Helix angle & $=$ & $\mathrm{H}_{\mathrm{A}}$ & $\mathrm{radians}$ & $\mathrm{M}^{0} \mathrm{~L}^{0} \mathrm{~T}^{0}$ & $=$ \\
Dry matter content & $=$ & $\mathrm{D}_{\mathrm{M}}$ & $\%$ & $\mathrm{M}^{0} \mathrm{~L}^{0} \mathrm{~T}^{0}$ & $=$ \\
Viscosity & Dynamic & $\mu$ & $\mathrm{Kg} / \mathrm{ms}$ & $\mathrm{ML}^{-1} \mathrm{~T}^{-1}$ & $\mathrm{~A}_{3}$ \\
\hline
\end{tabular}


Thus, a first-order regression model involving the dimensionless $\pi$-terms would first be investigated. The results of the first order regression gave the values of $\mathrm{R}^{2}$, predicted $\mathrm{R}^{2}$ and adjusted $\mathrm{R}^{2}$ to be 93.38\%, 90.23\%, 92.43\% for mass number, $97.83 \%$, $97.03 \%, 97.52 \%$ for power number and $89.64 \%$, $86.19 \%, 88.16 \%$ for processing time number which are desirable.

The result did not show any evidence of lack of fit and was used to locate the optimum.

Cassava chips weighing $25 \mathrm{~kg}, 30 \mathrm{~kg}, 35 \mathrm{~kg}, 40 \mathrm{~kg}$ and $45 \mathrm{~kg}$ each at different operational speed of 230,240 , 250, 260 and 270rpm was used for the experiment (25 runs). After each operation, the processing time was taken using a stop watch and the mass of the separated products weighed using a weighing balance. Their values were recorded for references. The power consumption of the motor during operation was measured using a clamp wattmeter. The individual test conditions and results of the experiment conducted are presented in Appendix A. The mathematical equation used for computing efficiency and throughput of the machine during the experiment are as follows [8];

$\mathrm{E}_{\mathrm{C}}=\frac{\mathrm{M}_{\mathrm{o}}}{\mathrm{M}_{\mathrm{i}}} \times 100$

$\mathrm{T}_{\mathrm{P}}=\frac{\mathrm{M}_{\mathrm{i}}}{\mathrm{t}} \times 3600$

where: $E_{q}$ is efficiency of machine in percentage (\%), $\mathrm{T}_{\mathrm{p}}$ is machine throughput in kilogram per hour
( $\mathrm{kg} / \mathrm{hr}$ ), $\mathrm{M}_{\mathrm{a}}$ is total mass of pellets recovered in $\mathrm{kg}$, $\mathrm{m}_{\mathrm{i}}$ is the mass of pre-conditioned cassava used in the experiment, $t$ is the processing time in second and $\mathrm{P}_{\mathrm{W}}$ is power consumed by the electric motor in watt.

\subsection{Results and Discussions}

The measured and predicted responses of the pelleting machine using the first-order are shown in Appendices A (Table A.1). The highest values for the measured mass number $\left(\mathrm{M}_{\mathrm{n}}\right)$, processing time number $\left(\mathrm{T}_{\mathrm{n}}\right)$, power consumption number $\left(\mathrm{P}_{\mathrm{Wn}}\right)$ (Appendix $\mathrm{A}$, Table A.1) are 38.73, 0.034 and 519696 with its corresponding predictive values of $31.89,0.032$ and 498296 respectively. Their corresponding residuals analyses of 6.84161, 0.00204 and 21400, percentage (\%) error values of $0.1766 \%, 0.06023 \%$ and $0.041 \%$ were obtained respectively. The compared residual analyses and percentage (\%) errors of the responses at maximum values at both cases were close to negligible values. The predicted and measured values of the centrifuge responses were compared (Figure 2). Figure 2 was plotted using the measured and predicted values of the responses shown in Appendices A (Table A.1). There are positive linear relationships between the predicted and measured values of the responses respectively. A good agreement between measured and predicted values was found with high coefficient of determination $\left(\mathrm{R}^{2}\right)$.

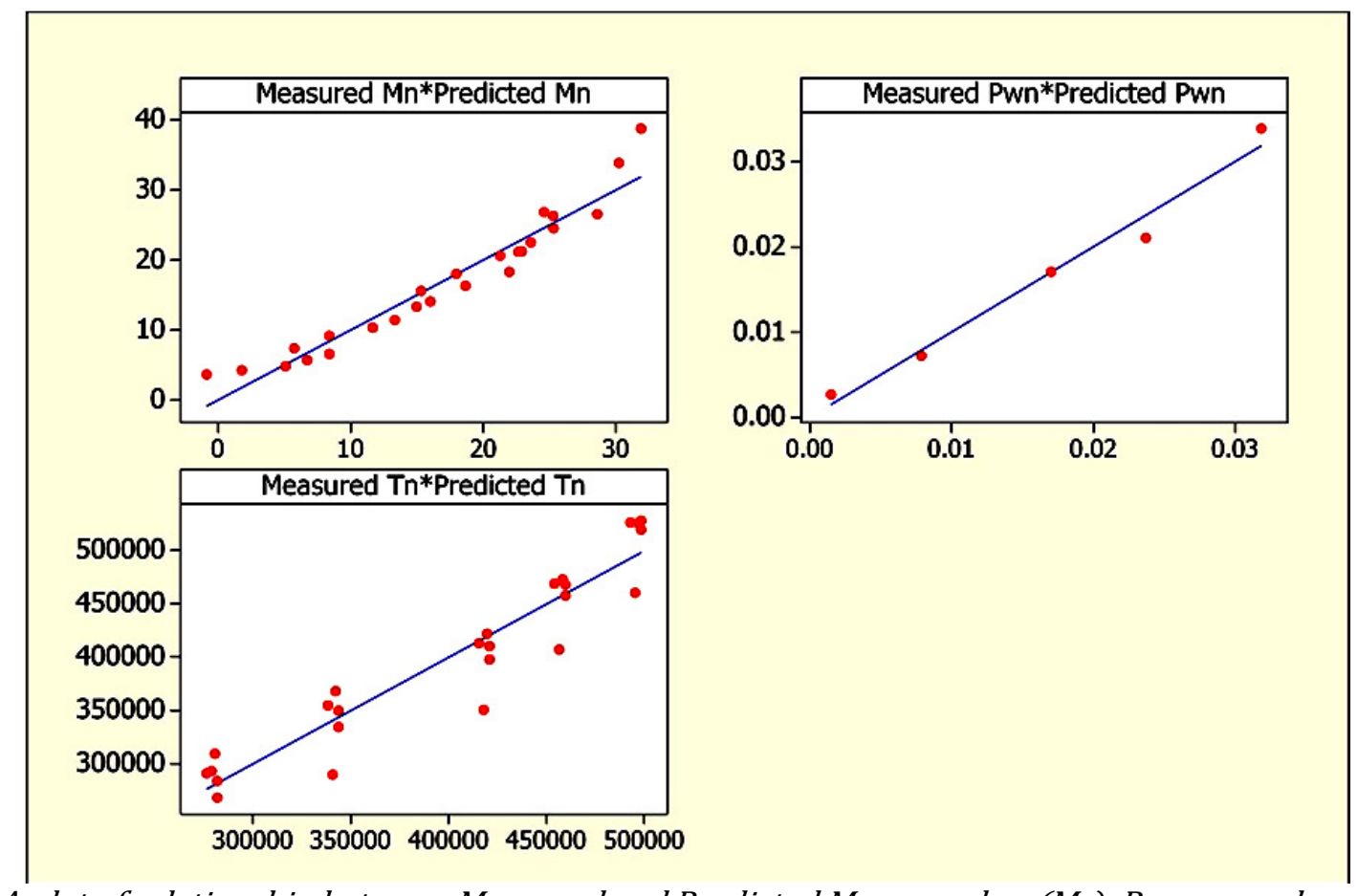

Figure 2: A plot of relationship between Measured and Predicted Mass number $\left(M_{n}\right)$, Power number $\left(P_{w n}\right)$, and Processing time number $\left(T_{n}\right)$ 
Hence, the empirical models developed (equations 11 - 16) are reasonably accurate since the results of the actual values of the responses are within 95\% prediction interval. The $95 \%$ prediction interval is the range in which any individual value (predicted) is statistically expected to fall within. Therefore, the models developed were used to determine the optimal setting of the centrifuge performance parameters.

\section{OPTIMIZATION OF THE DEVELOPED RESPONSE SURFACE MODELS}

The models developed for the performance parameters of the Pelleting machine were optimized using Minitab optimization facility known as response optimizer. The facility provides you with an optimal solution for the input variable combinations and an optimization plot. The calculated dimensionless parameters such as helix angle, density number and dry matter content are coded as $\mathrm{X}_{1}, \mathrm{X}_{2}$ and $\mathrm{X}_{3}$, respectively were used as the input variables for the regression. The minimum and maximum values of $X_{1}$, $Y_{1}, Y_{2}$ and $Y_{3}$ variables of the dimensionless parameters were obtained as follows;

$$
\begin{aligned}
& \mathrm{X}_{1 \text { min }}=\frac{\mu_{\min }}{D_{\max }^{2} C_{S \max } \rho_{\max }} \\
& \mathrm{X}_{1 \text { max }}=\frac{\mu_{\max }}{\mathrm{D}_{\min }^{2} \mathrm{C}_{\mathrm{Smin}} \rho_{\min }} \\
& \mathrm{Y}_{1 \text { min }}=\frac{\mathrm{M}_{\mathrm{o}_{\text {min }}}}{\mathrm{D}_{\max }^{3} \rho_{\max }} \\
& \mathrm{Y}_{1 \text { max }}=\frac{\mathrm{M}_{\mathrm{omax}_{\text {max }}}}{\mathrm{D}_{\text {min }}^{3} \rho_{\text {min }}} \\
& \mathrm{Y}_{2 \min }=\frac{\mathrm{P}_{\mathrm{W}_{\text {min }}}}{\mathrm{D}_{\text {max }}^{5} \mathrm{C}_{\mathrm{S}}^{3} \rho_{\max }} \\
& \mathrm{Y}_{2 \text { max }}=\frac{\mathrm{P}_{\mathrm{W}_{\text {max }}}}{\mathrm{D}_{\text {min }}^{5} \mathrm{C}_{\mathrm{S}}^{3} \rho_{\text {min }}} \\
& \mathrm{Y}_{3_{\text {min }}}=\mathrm{t}_{\text {min }} \mathrm{C}_{\text {Smin }} \\
& \mathrm{Y}_{3_{\max }}=\mathrm{t}_{\max } \mathrm{C}_{\text {Smax }}
\end{aligned}
$$

\subsection{Contour Plots}

Contour plots are two dimensional and are useful for establishing operating conditions that produce desirable response values. The plot is used in combination with the optimization plot to find the best operating conditions for maximizing and minimizing the responses. The white regions on the plots are the feasible regions or areas that satisfy the criteria for all responses. In figure 3 , the white region shows the range of $\mathrm{x}_{1}(0.004$ to 0.0125$)$ and $\mathrm{x}_{2}$ (0.0 to $60)$ where the criteria for both response variables are satisfied. The optimal settings for the responses are expected to fall within this area.

\subsection{Optimization of the Machine Performance}

In order to determine the optimal machine performance, constraints and the objective functions for the dependent variables are defined and the best values of input parameters are determined. The objective functions for the three dependent variables are;

1. Maximize Mass number $\left(\mathrm{M}_{\mathrm{n}}\right)$

2. Minimize Power number $\left(\mathrm{P}_{\mathrm{Wn}}\right)$

3. Minimize processing time number $\left(\mathrm{T}_{\mathrm{n}}\right)$

Subject to the following constraints obtained from equations (19-26);

1. $6.04 \leq$ Mass number $\leq 52.42$

2. $0.0014 \leq$ Power number $\leq 0.067$

3. $228390 \leq$ Processing time number $\leq 617544$ The optimal results from the response optimizer are based on the developed models.
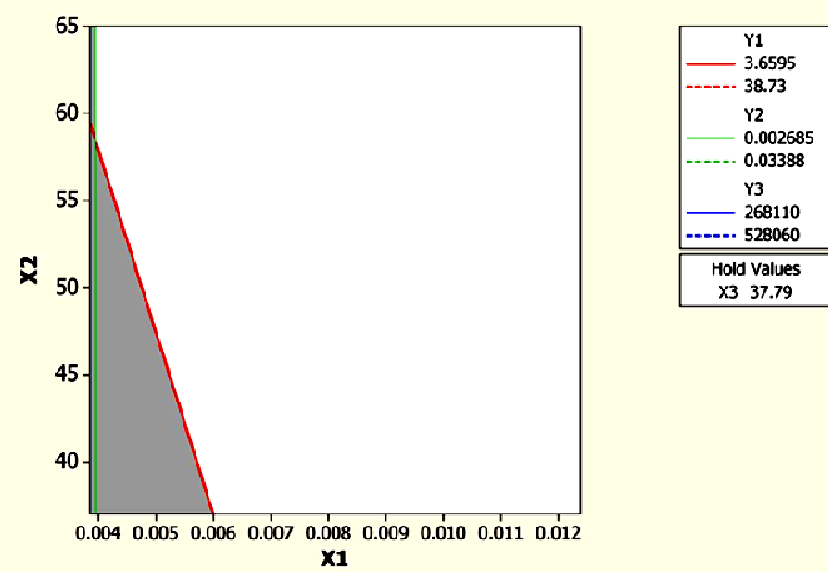

Figure 3: Contour plots of responses $Y_{1}, Y_{2}$ and $Y_{3}$

Table 2: Result of response optimizer for minimizing two responses and maximizing one response

\begin{tabular}{lccccc}
\hline Goal & Lower & Target & Upper & Weight & Import \\
\hline Y1 & 3 & 52 & 52 & 1 & 1 \\
$\begin{array}{l}\text { Maximum } \\
\text { Y2 }\end{array}$ & 0 & 0 & 0 & 1 & 1 \\
$\begin{array}{l}\text { Minimum } \\
\text { Y3 }\end{array}$ & 228390 & 118390 & 617544 & 1 & 1 \\
Minimum & & & & &
\end{tabular}

Starting Point

$\mathrm{X} 1=0.0104$

$\mathrm{X} 2=51$

$\mathrm{X} 3=37.79$

Global Solution

$\mathrm{X} 1=0.0123876$

$\mathrm{X} 2=37$

$\mathrm{X} 3=33.83$ 
Predicted Responses

$\mathrm{Y} 1=29, \quad$ desirability $=0.518970$

$\mathrm{Y} 2=0.03, \quad$ desirability $=0.565673$

$\mathrm{Y} 3=273220$, desirability $=0.884801$

Composite Desirability $=0.638045$

The optimal values for mass number, power number and processing time number are $29,0.03 \%$, and 273220 respectively. The optimal values of efficiency, throughput and power consumed were calculated using equations (17), (18) and (15) respectively. The individual desirability and composited desirability fall within the acceptable range of 0 (zero if one or more product characteristics are unacceptable) to 1 ( 1 if all product characteristics are on target). The efficiency, throughput and power consumed were calculated using values in Appendix A (Table A.2) as reference values.

Equation 27 was used to calculate the optimal value of power consumed by the machine and it was derived by equating equations 14 and 15 .

$\mathrm{P}_{\mathrm{W}}=\frac{\mathrm{M}_{\mathrm{o}} \mathrm{Y}_{2} \mathrm{D}^{2} \mathrm{C}_{\mathrm{S}}^{3}}{\mathrm{Y}_{1}}$

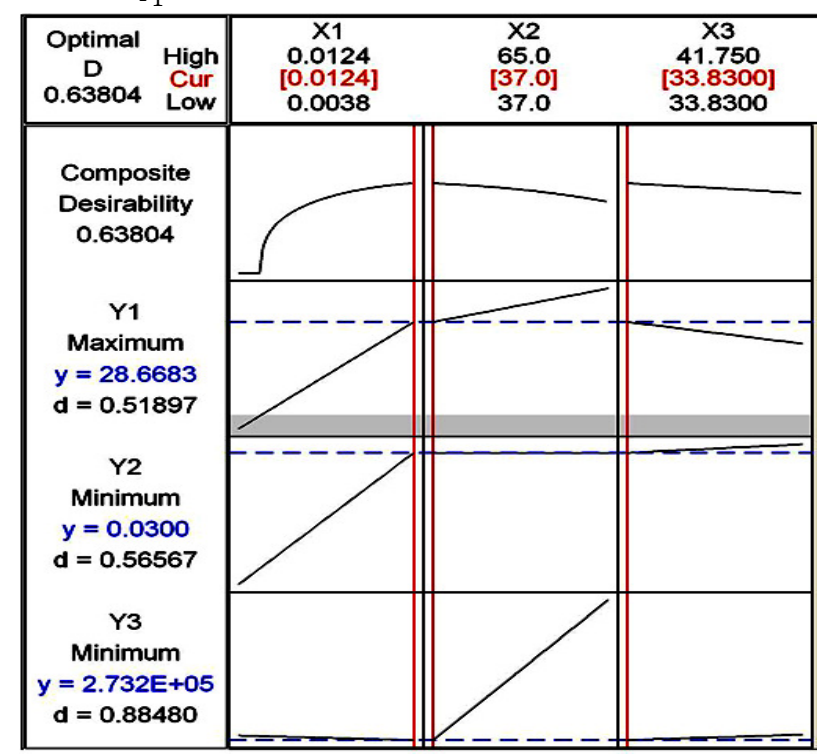

Figure 4: The result of response optimizer for

minimizing two responses and maximizing one response

The optimal values of efficiency, throughput and power consumed are $58 \%, 75.76 \mathrm{~kg} / \mathrm{hr}$ and $4106.36 \mathrm{~W}$. The corresponding optimal values of the input parameters that gave these optimal responses are density number (0.0104), helix angle $\left(37^{\circ}\right)$ and dry matter content $(33.83 \%)$.
The optimal size and the correct variable combinations have been successfully determined using the optimization tool of the RSM.

\section{CONCLUSIONS}

The study was conducted to obtain a statistically wellordered data set to understand the main effects of key process parameters on the performance of the Pelleting machine. Critical to this investigation is to maximize the throughput, efficiency and minimize the power consumption. The models were simulated by systematically varying desired process parameters to study their main effects on the 3 process responses. The optimal result of the responses efficiency, throughput and power consumption of the machine were determined as $58 \%, 75.76 \mathrm{~kg} / \mathrm{hr}$ and 4106.36W.respectively.

\section{REFERENCES}

1. Ashaye O., Adebayo, S., Oladimeji, L, Arowosafe, B E., (2010). Physiochemical, Rheological and Consumer Acceptability of Cassava Starch Salad Cream. Journal of American Science, 6(1):pp 65-72, Marsland Press.

2. Crowley, P.R. (199) Transferring LEC technology to developing countries; from concept to application and beyond. In Low cost Extrusion Cookers, second international workshop proceeding, Colorado state University, Ft Collins, Co, pp 11-14.

3. Farrel, D. (2001) Extrusion equipment, types, function and application. Symposium on extrusion process and product development, American Association of cereal chemist, St Paul MN.

4. Kasprzak, W, Lysik and Rybaczuk, M(1990) Dimensional Analysis in the Identification of Mathematical models. World scientific Publishing Co. Plc. Ltd. Singapore.

5. Langhaar, H.L (1951) Dimensional Analysis and Theory of Models, John Wiley and Sons. Inc., New York.

6. Murphy, G (1950) Similitude in Engineering. The Ronald Press Company, New York.

7. Oyewole, O.B and Obieze, N. (1995). Processing and Characteristics of Tapioca Meal from Cassava. Tropical Science 35:pp 19-22.

8. Ugoamadi C.C (2012) Development of a Cassava Pelleting Machine. Nigerian Journal of Technology, Vol. 31, No.3:pp 233-240. 


\section{APPENDIX A:}

Table A.1: Verification of Measured and Predicted Responses of the Pelleting Machine (First-Order)

\begin{tabular}{|c|c|c|c|c|c|c|c|c|c|c|c|}
\hline $\begin{array}{c}\text { Measured } \\
M_{n}\end{array}$ & $\begin{array}{c}\text { Predicted } \\
M_{n}\end{array}$ & Residual & $\%$ error & $\begin{array}{c}\text { Measured } \\
\text { Pwn }\end{array}$ & $\begin{array}{l}\text { Predicted } \\
\text { Pwn }\end{array}$ & Residual & $\begin{array}{c}\% \\
\text { error }\end{array}$ & $\begin{array}{c}\text { Measured } \\
\text { Tn }\end{array}$ & $\begin{array}{l}\text { Predicted } \\
\text { Tn }\end{array}$ & Residual & $\begin{array}{l}\% \\
\text { error }\end{array}$ \\
\hline 3.6595 & -0.8682 & 4.5276 & 1.2372 & 0.00268 & 0.00147 & 0.00120 & 0.4493 & 309396 & 280425 & 28970 & 0.0936 \\
\hline 4.2399 & 1.7709 & 2.4690 & 0.5823 & 0.00268 & 0.00147 & 0.00120 & 0.4493 & 368046 & 342270 & 25776 & 0.0700 \\
\hline 4.7699 & 5.0699 & -0.299 & -0.062 & 0.00268 & 0.00147 & 0.00120 & 0.4493 & 421866 & 419575 & 2291.2 & 0.0054 \\
\hline 5.6533 & 6.7194 & -1.066 & -0.188 & 0.00268 & 0.00147 & 0.00120 & 0.4493 & 473064 & 458227 & 14836. & 0.0313 \\
\hline 6.5871 & 8.3688 & -1.781 & -0.270 & 0.00268 & 0.00147 & 0.00120 & 0.4493 & 526056 & 496880 & 29176. & 0.0554 \\
\hline 7.3939 & 5.7246 & 1.6692 & 0.2257 & 0.00721 & 0.00787 & -0.0006 & -0.091 & 293472 & 278852 & 14620 . & 0.0498 \\
\hline 9.1684 & 8.3638 & 0.8046 & 0.0877 & 0.00721 & 0.00787 & -0.0006 & -0.091 & 289584 & 340696 & -51111 & -0.176 \\
\hline 10.3514 & 11.662 & -1.311 & -0.126 & 0.00721 & 0.00787 & -0.0006 & -0.091 & 350784 & 418001 & -67217 & -0.191 \\
\hline 11.4359 & 13.312 & -1.876 & -0.164 & 0.00721 & 0.00787 & -0.0006 & -0.091 & 407232 & 456654 & -49421 & -0.121 \\
\hline 13.3082 & 14.960 & -1.652 & -0.124 & 0.00721 & 0.0078 & -0.0006 & -0.091 & 460368 & 495306 & -34938 & -0.075 \\
\hline 15.5729 & 15.324 & 0.2482 & 0.0159 & 0.01707 & 0.01702 & 0.00005 & 0.0029 & 291000 & 276406 & 14594 & 0.0501 \\
\hline 18.0462 & 17.963 & 0.0824 & 0.0045 & 0.01707 & 0.01702 & 0.00005 & 0.0029 & 354750 & 338250 & 16500. & 0.0465 \\
\hline 20.6112 & 21.262 & -0.651 & -0.031 & 0.01707 & 0.0170 & 0.00005 & 0.0029 & 413250 & 415555 & -2305 & -0.005 \\
\hline 21.2524 & 22.91 & -1.659 & -0.078 & 0.01707 & 0.01702 & 0.00005 & 0.0029 & 468900 & 454208 & 14692 & 0.0313 \\
\hline 26.8404 & 24.561 & 2.2786 & 0.0849 & 0.01707 & 0.01702 & 0.00005 & 0.0029 & 526500 & 492860 & 33639. & 0.0638 \\
\hline 14.0883 & 16.000 & -1.912 & -0.135 & 0.02104 & 0.02368 & -0.0026 & -0.125 & 284076 & 281842 & 2234.1 & 0.0078 \\
\hline 16.2912 & 18.640 & -2.348 & -0.144 & 0.02104 & 0.02368 & -0.0026 & -0.125 & 349908 & 343686 & 6221.9 & 0.0177 \\
\hline 18.2892 & 21.938 & -3.649 & -0.199 & 0.02104 & 0.02368 & -0.0026 & -0.125 & 410436 & 420991 & -10555 & -0.025 \\
\hline 22.5413 & 23.588 & -1.047 & -0.046 & 0.02104 & 0.02368 & -0.0026 & -0.125 & 468312 & 459644 & 8668.1 & 0.0185 \\
\hline 26.2811 & 25.237 & 1.0432 & 0.0397 & 0.02104 & 0.02368 & -0.0026 & -0.125 & 528060 & 498296 & 29763. & 0.0563 \\
\hline 21.1681 & 22.654 & -1.486 & -0.070 & 0.03388 & 0.03183 & 0.00204 & 0.0602 & 268110 & 281841 & -13731 & -0.051 \\
\hline 24.5089 & 25.293 & -0.784 & -0.032 & 0.03388 & 0.03184 & 0.00204 & 0.0602 & 334692 & 343685 & -8993 & -0.026 \\
\hline 26.5513 & 28.59 & -2.040 & -0.076 & 0.03388 & 0.03184 & 0.00204 & 0.0602 & 397872 & 420991 & -23118 & -0.058 \\
\hline 33.8456 & 30.241 & 3.6038 & 0.1064 & 0.03388 & 0.03184 & 0.00204 & 0.0602 & 457650 & 459643 & -1993 & -0.004 \\
\hline 38.7328 & 31.891 & 6.8416 & 0.17664 & 0.03388 & 0.03184 & 0.00204 & 0.060 & 519696 & 498296 & 21400 & 0.041 \\
\hline
\end{tabular}

where $\mathrm{M}_{\mathrm{n}}=$ mass number, $\mathrm{P}_{\mathrm{wn}}=$ power consumption number, $\mathrm{T}_{\mathrm{n}}=$ processing time number

Table A.2: Reference Value used for calculating the optimal values of the responses

\begin{tabular}{ll}
\hline Reference Value & Values \\
\hline Dry matter (\%) & 34 \\
Starch content (\%) & 18.3 \\
Bulk density & 1174.02 \\
$\left(\mathrm{~kg} / \mathrm{m}^{3}\right)$ & 23.383 \\
Viscosity $(\mathrm{kg} / \mathrm{ms})$ & 0.15 \\
screw conveyor pitch (m) & 25 \\
Mass of pre-conditioned cassava $\mathrm{M}_{\mathrm{i}}(\mathrm{kg})$ & 14.50 \\
Mass of Pellets formed $\mathrm{M}_{\mathrm{o}}(\mathrm{kg})$ & 1345.2 \\
Processing time t $(\mathrm{sec})$ & 230 \\
Speed (RPM) & \\
\hline
\end{tabular}

